\title{
Weighted boundedness of multilinear singular integral operator with general kernels for the extreme cases
}

\section{Mingjun Zhang and Yanfeng Guo*}

"Correspondence: guoyan_feng@163.com College of Science, Guangxi University of Science and Technology, Liuzhou, 545006, P.R. China

\begin{abstract}
We prove the weighted boundedness properties for the multilinear operator associated to the singular integral operator with general kernels for the extreme cases. MSC: 42B20; 42B25
\end{abstract}

Keywords: multilinear operator; singular integral operator; BMO space

\section{Introduction and preliminaries}

As for the development of singular integral operators, their commutators and multilinear operators have been well studied (see [1-9]). Let $T$ be the Calderón-Zygmund singular integral operator and $b \in B M O\left(R^{n}\right)$, a classical result of Coifman et al. (see [5]) stated that the commutator $[b, T](f)=T(b f)-b T(f)$ is bounded on $L^{p}\left(R^{n}\right)$ for $1<p<\infty$. In [10], the authors obtain the boundedness properties of the commutators for the extreme values of $p$ (that is, $p=1$ and $p=\infty$ ). Note that $[b, T]$ is not bounded for the end point boundedness. The purpose of this paper is to introduce some multilinear operator associated to the singular integral operator with general kernels (see [11]) and prove the weighted boundedness properties of the multilinear operators for the extreme cases.

First, let us introduce some preliminaries (see $[6,9])$. Throughout this paper, $Q$ will denote a cube of $R^{n}$ with sides parallel to the axes. For a locally integrable functions $b$ and a weight function $w$ (that is, a non-negative locally integrable function), let $w(Q)=$ $\int_{Q} w(x) d x, w_{Q}=|Q|^{-1} \int_{Q} w(x) d x$, the weighted sharp function of $b$ is defined by

$$
b^{\#}(x)=\sup _{Q \ni x} \frac{1}{w(Q)} \int_{Q}\left|b(y)-b_{Q}\right| w(y) d y .
$$

We say that $b$ belongs to $B M O(w)$ if $b^{\#}$ belongs to $L^{\infty}(w)$, and we define $\|b\|_{B M O(w)}=$ $\left\|b^{\#}\right\|_{L^{\infty}(w)}$. If $w=1$, we denote $B M O(w)=B M O\left(R^{n}\right)$. It has been known that (see [9])

$$
\left\|b-b_{2^{k} Q}\right\|_{B M O} \leq C k\|b\|_{B M O}
$$


We also define the central $B M O$ space by $C M O\left(R^{n}\right)$, which is the space of those functions $f \in L_{\text {loc }}\left(R^{n}\right)$ such that

$$
\|f\|_{C M O}=\sup _{r>1}|Q(0, r)|^{-1} \int_{Q}\left|f(x)-f_{Q}\right| d x<\infty .
$$

It is well known that (see $[6,9])$

$$
\|f\|_{C M O} \approx \sup _{r>1} \inf _{c \in C}|Q(0, r)|^{-1} \int_{Q}|f(x)-c| d x .
$$

Definition 1 Let $1<p<\infty$ and $w$ be a non-negative weight functions on $R^{n}$. We shall call $B_{p}(w)$ the space of those functions $f$ on $R^{n}$ such that

$$
\|f\|_{B_{p}(w)}=\sup _{r>1}[w(Q(0, r))]^{-1 / p}\left\|f \chi_{Q(0, r)}\right\|_{L^{p}(w)}<\infty .
$$

The $A_{p}$ weight is defined by (see [6])

$$
\begin{aligned}
A_{p} & =\left\{0<w \in L_{\mathrm{loc}}^{1}\left(R^{n}\right): \sup _{Q}\left(\frac{1}{|Q|} \int_{Q} w(x) d x\right)\left(\frac{1}{|Q|} \int_{Q} w(x)^{-1 /(p-1)} d x\right)^{p-1}<\infty\right\}, \\
1 & <p<\infty,
\end{aligned}
$$

and

$$
A_{1}=\left\{0<w \in L_{\mathrm{loc}}^{1}\left(R^{n}\right): \sup _{Q \ni x} \frac{1}{|Q|} \int_{Q} w(y) d y \leq C w(x) \text {, a.e. }\right\} .
$$

\section{Theorems}

In this paper, we will study the following multilinear singular integral operator (see [11]).

Definition 2 Let $T: S \rightarrow S^{\prime}$ be a linear operator such that $T$ is bounded on $L^{2}\left(R^{n}\right)$ and has a kernel $K$, that is, there exists a locally integrable function $K(x, y)$ on $R^{n} \times R^{n} \backslash\{(x, y) \in$ $R^{n} \times R^{n}: x=y$ s such that

$$
T(f)(x)=\int_{R^{n}} K(x, y) f(y) d y
$$

for every bounded and compactly supported function $f$, where $K$ satisfies

$$
\begin{aligned}
& |K(x, y)| \leq C|x-y|^{-n}, \\
& \int_{2|y-z|<|x-y|}(|K(x, y)-K(x, z)|+|K(y, x)-K(z, x)|) d x \leq C,
\end{aligned}
$$

and there is a sequence of positive constant numbers $\left\{C_{k}\right\}$ such that for any $k \geq 1$,

$$
\begin{aligned}
& \left(\int_{2^{k}|z-y| \leq|x-y|<2^{k+1}|z-y|}(|K(x, y)-K(x, z)|+|K(y, x)-K(z, x)|)^{q} d y\right)^{1 / q} \\
& \quad \leq C_{k}\left(2^{k}|z-y|\right)^{-n / q^{\prime}}
\end{aligned}
$$

where $1<q^{\prime}<2$ and $1 / q+1 / q^{\prime}=1$. 
Let $m_{j}$ be the positive integers $(j=1, \ldots, l), m_{1}+\cdots+m_{l}=m$ and $b_{j}$ be the functions on $R^{n}(j=1, \ldots, l)$. Set, for $1 \leq j \leq l$,

$$
R_{m_{j}+1}\left(b_{j} ; x, y\right)=b_{j}(x)-\sum_{|\alpha| \leq m_{j}} \frac{1}{\alpha !} D^{\alpha} b_{j}(y)(x-y)^{\alpha} .
$$

The multilinear operator associated to $T$ is defined by

$$
T_{b}(f)(x)=\int_{R^{n}} \frac{\prod_{j=1}^{l} R_{m_{j}+1}\left(b_{j} ; x, y\right)}{|x-y|^{m}} K(x, y) f(y) d y .
$$

Note that the classical Calderón-Zygmund singular integral operator satisfies Definition 2 (see $[7,8]$ ). Also note that when $m=0, T_{b}$ is just a multilinear commutator of $T$ and $b$ (see [1-3]). It is well known that a multilinear operator, as a non-trivial extension of a commutator, is of great interest in harmonic analysis and has been widely studied by many authors (see [1-3]). In this paper, we will study the weighted boundedness properties of the multilinear operators $T_{b}$ for the extreme cases (see [12-15]).

We shall prove the following theorems in Section 3.

Theorem 1 Let $T$ be the singular integral operator as Definition 2, and we have the sequence $\left\{k^{m} C_{k}\right\} \in l^{1}, w \in A_{1}$ and $D^{\alpha} b_{j} \in B M O\left(R^{n}\right)$ for all $\alpha$ with $|\alpha|=m_{j}$ and $j=1, \ldots, l$. Then $T_{b}$ is bounded from $L^{\infty}(w)$ to $B M O(w)$.

Theorem 2 Let $T$ be the singular integral operator as Definition 2, and we have the sequence $\left\{k^{m} C_{k}\right\} \in l^{1}, 1<p<\infty, w \in A_{1}$, and $D^{\alpha} b_{j} \in B M O\left(R^{n}\right)$ for all $\alpha$ with $|\alpha|=m_{j}$ and $j=1, \ldots, l$. Then $T_{b}$ is bounded from $B_{p}(w)$ to $C M O(w)$.

\section{Proofs of theorems}

We begin with two preliminaries lemmas.

Lemma 1 (see [3]) Let b be a function on $R^{n}$ and $D^{\alpha} b \in L^{q}\left(R^{n}\right)$ for $|\alpha|=m$ and some $q>n$. Then

$$
\left|R_{m}(b ; x, y)\right| \leq C|x-y|^{m} \sum_{|\alpha|=m}\left(\frac{1}{|\tilde{Q}(x, y)|} \int_{\tilde{Q}(x, y)}\left|D^{\alpha} b(z)\right|^{q} d z\right)^{1 / q},
$$

where $\tilde{Q}(x, y)$ is the cube centered at $x$ and having side length $5 \sqrt{n}|x-y|$.

Lemma 2 (see [11]) Let $T$ be the singular integral operator as Definition 2, and we have the sequence $\left\{C_{k}\right\} \in l^{1}$. Then $T$ is bounded on $L^{p}\left(R^{n}, w\right)$ for $w \in A_{p}$ with $1<p<\infty$.

Proof of Theorem 1 It is only for us to prove that there exists a constant $C_{Q}$ such that

$$
\frac{1}{w(Q)} \int_{Q}\left|T_{b}(f)(x)-C_{Q}\right| w(x) d x \leq C\|f\|_{L^{\infty}(w)}
$$

holds for any cube $Q$. Without loss of generality, we may assume $l=2$. Fix a cube $Q=Q\left(x_{0}, d\right)$. Let $\tilde{Q}=5 \sqrt{n} Q$ and $\tilde{b}_{j}(x)=b_{j}(x)-\sum_{|\alpha|=m} \frac{1}{\alpha !}\left(D^{\alpha} b_{j}\right) \tilde{Q}^{x^{\alpha}}$, then $R_{m}\left(b_{j} ; x, y\right)=$ 
$R_{m}\left(\tilde{b}_{j} ; x, y\right)$ and $D^{\alpha} \tilde{b}_{j}=D^{\alpha} b_{j}-\left(D^{\alpha} b_{j}\right) \tilde{Q}$ for $|\alpha|=m_{j}$. We write, for $f_{1}=f \chi_{\tilde{Q}}$ and $f_{2}=f \chi_{R^{n} \backslash \tilde{Q}}$,

$$
\begin{aligned}
T_{b}(f)(x)= & \int_{R^{n}} \frac{\prod_{j=1}^{2} R_{m_{j}+1}\left(\tilde{b}_{j} ; x, y\right)}{|x-y|^{m}} K(x, y) f(y) d y \\
= & \int_{R^{n}} \frac{\prod_{j=1}^{2} R_{m_{j}}\left(\tilde{b}_{j} ; x, y\right)}{|x-y|^{m}} K(x, y) f_{1}(y) d y \\
& -\sum_{\left|\alpha_{1}\right|=m_{1}} \frac{1}{\alpha_{1} !} \int_{R^{n}} \frac{R_{m_{2}}\left(\tilde{b}_{2} ; x, y\right)(x-y)^{\alpha_{1}} D^{\alpha_{1}} \tilde{b}_{1}(y)}{|x-y|^{m}} K(x, y) f_{1}(y) d y \\
& -\sum_{\left|\alpha_{2}\right|=m_{2}} \frac{1}{\alpha_{2} !} \int_{R^{n}} \frac{R_{m_{1}}\left(\tilde{b}_{1} ; x, y\right)(x-y)^{\alpha_{2}} D^{\alpha_{2}} \tilde{b}_{2}(y)}{|x-y|^{m}} K(x, y) f_{1}(y) d y \\
& +\sum_{\left|\alpha_{1}\right|=m_{1},\left|\alpha_{2}\right|=m_{2}} \frac{1}{\alpha_{1} ! \alpha_{2} !} \int_{R^{n}} \frac{(x-y)^{\alpha_{1}+\alpha_{2}} D^{\alpha_{1}} \tilde{b}_{1}(y) D^{\alpha_{2}} \tilde{b}_{2}(y)}{|x-y|^{m}} K(x, y) f_{1}(y) d y \\
& +\int_{R^{n}} \frac{\prod_{j=1}^{2} R_{m_{j}+1}\left(\tilde{b}_{j} ; x, y\right)}{|x-y|^{m}} K(x, y) f_{2}(y) d y \\
= & T\left(\frac{\prod_{j=1}^{2} R_{m_{j}}\left(\tilde{b}_{j} ; x, \cdot\right)}{|x-\cdot|^{m}} f_{1}\right) \\
& -T\left(\sum_{\left|\alpha_{1}\right|=m_{1}} \frac{1}{\alpha_{1} !} \frac{R_{m_{2}}\left(\tilde{b}_{2} ; x, \cdot\right)(x-\cdot)^{\alpha_{1}} D^{\alpha_{1}} \tilde{b}_{1}}{|x-\cdot|^{m}} f_{1}\right) \\
& -T\left(\sum_{\left|\alpha_{2}\right|=m_{2}} \frac{1}{\alpha_{2} !} \frac{R_{m_{1}}\left(\tilde{b}_{1} ; x, \cdot\right)(x-\cdot)^{\alpha_{2}} D^{\alpha_{2}} \tilde{b}_{2}}{|x-\cdot|^{m}} f_{1}\right) \\
& +T\left(\sum_{\left|\alpha_{1}\right|=m_{1},\left|\alpha_{2}\right|=m_{2}} \frac{1}{\alpha_{1} ! \alpha_{2} !} \frac{(x-\cdot)^{\alpha_{1}+\alpha_{2}} D^{\alpha_{1}} \tilde{b}_{1} D^{\alpha_{2}} \tilde{b}_{2}}{|x-\cdot|^{m}} f_{1}\right) \\
& T\left(\frac{\prod_{j=1}^{2} R_{m_{j}+1}\left(\tilde{b}_{j} ; x, \cdot\right)}{|x-\cdot|^{m}} f_{2}\right), \\
& \\
&
\end{aligned}
$$

then

$$
\begin{aligned}
\left|T_{b}(f)(x)-T_{\tilde{b}}\left(f_{2}\right)\left(x_{0}\right)\right| \leq & \left|T\left(\frac{\prod_{j=1}^{2} R_{m_{j}}\left(\tilde{b}_{j} ; x, \cdot\right)}{|x-\cdot|^{m}} f_{1}\right)\right| \\
& +\left|T\left(\sum_{\left|\alpha_{1}\right|=m_{1}} \frac{1}{\alpha_{1} !} \frac{R_{m_{2}}\left(\tilde{b}_{2} ; x, \cdot\right)(x-\cdot)^{\alpha_{1}} D^{\alpha_{1}} \tilde{b}_{1}}{|x-\cdot|^{m}} f_{1}\right)\right| \\
& +\left|T\left(\sum_{\left|\alpha_{2}\right|=m_{2}} \frac{1}{\alpha_{2} !} \frac{R_{m_{1}}\left(\tilde{b}_{1} ; x, \cdot\right)(x-\cdot)^{\alpha_{2}} D^{\alpha_{2}} \tilde{b}_{2}}{|x-\cdot|^{m}} f_{1}\right)\right| \\
& +\left|T\left(\sum_{\left|\alpha_{1}\right|=m_{1},\left|\alpha_{2}\right|=m_{2}} \frac{1}{\alpha_{1} ! \alpha_{2} !} \frac{(x-\cdot)^{\alpha_{1}+\alpha_{2}} D^{\alpha_{1}} \tilde{b}_{1} D^{\alpha_{2}} \tilde{b}_{2}}{|x-\cdot|^{m}} f_{1}\right)\right| \\
& +\left|T_{\tilde{b}}\left(f_{2}\right)(x)-T_{\tilde{b}}\left(f_{2}\right)\left(x_{0}\right)\right| \\
= & I_{1}(x)+I_{2}(x)+I_{3}(x)+I_{4}(x)+I_{5}(x)
\end{aligned}
$$


and

$$
\begin{aligned}
& \frac{1}{w(Q)} \int_{Q}\left|T_{b}(f)(x)-T_{\tilde{b}}\left(f_{2}\right)\left(x_{0}\right)\right| w(x) d x \\
& \leq \frac{1}{w(Q)} \int_{Q} I_{1}(x) w(x) d x+\frac{1}{w(Q)} \int_{Q} I_{2}(x) w(x) d x+\frac{1}{w(Q)} \int_{Q} I_{3}(x) w(x) d x \\
& \quad+\frac{1}{w(Q)} \int_{Q} I_{4}(x) w(x) d x+\frac{1}{w(Q)} \int_{Q} I_{5}(x) w(x) d x \\
& \quad=I_{1}+I_{2}+I_{3}+I_{4}+I_{5} .
\end{aligned}
$$

Now, let us estimate $I_{1}, I_{2}, I_{3}, I_{4}$, and $I_{5}$, respectively. First, for $x \in Q$ and $y \in \tilde{Q}$, by Lemma 1 , we get

$$
R_{m}\left(\tilde{b}_{j} ; x, y\right) \leq C|x-y|^{m} \sum_{\left|\alpha_{j}\right|=m}\left\|D^{\alpha_{j}} b_{j}\right\|_{B M O}
$$

thus, by the $L^{p}(w)$-boundedness of $T$ for $1<p<\infty$ (Lemma 2) and Hölder's inequality, we obtain

$$
\begin{aligned}
I_{1} & \leq C \prod_{j=1}^{2}\left(\sum_{\left|\alpha_{j}\right|=m_{j}}\left\|D^{\alpha_{j}} b_{j}\right\|_{B M O}\right) \frac{1}{w(Q)} \int_{Q}\left|T\left(f_{1}\right)(x)\right| w(x) d x \\
& \leq C \prod_{j=1}^{2}\left(\sum_{\left|\alpha_{j}\right|=m_{j}}\left\|D^{\alpha_{j}} b_{j}\right\|_{B M O}\right)\left(\frac{1}{w(Q)} \int_{R^{n}}\left|T\left(f_{1}\right)(x)\right|^{p} w(x) d x\right)^{1 / p} \\
& \leq C \prod_{j=1}^{2}\left(\sum_{\left|\alpha_{j}\right|=m_{j}}\left\|D^{\alpha_{j}} b_{j}\right\|_{B M O}\right)\left(\frac{1}{w(Q)} \int_{R^{n}}\left|f_{1}(x)\right|^{p} w(x) d x\right)^{1 / p} \\
& \leq C \prod_{j=1}^{2}\left(\sum_{\left|\alpha_{j}\right|=m_{j}}\left\|D^{\alpha_{j}} b_{j}\right\|_{B M O}\right)\left(\frac{w(\tilde{Q})}{w(Q)}\right)^{1 / p}\|f\|_{L^{\infty}(w)} \\
& \leq C \prod_{j=1}^{2}\left(\sum_{\left|\alpha_{j}\right|=m_{j}}\left\|D^{\alpha_{j}} b_{j}\right\|_{B M O}\right)\|f\|_{L^{\infty}(w) .}
\end{aligned}
$$

For $I_{2}$, since $w \in A_{1}, w$ satisfies the reverse of Hölder's inequality:

$$
\left(\frac{1}{|Q|} \int_{Q} w(x)^{p_{0}} d x\right)^{1 / p_{0}} \leq \frac{C}{|Q|} \int_{Q} w(x) d x
$$

for all cube $Q$ and some $1<p_{0}<\infty$ (see [13]), thus, by the $L^{p}$-boundedness of $T$ for $p>1$, we get

$$
\begin{aligned}
I_{2} & \leq C \sum_{\left|\alpha_{2}\right|=m_{2}}\left\|D^{\alpha_{2}} b_{2}\right\|_{B M O} \sum_{\left|\alpha_{1}\right|=m_{1}} \frac{1}{w(Q)} \int_{Q}\left|T\left(D^{\alpha_{1}} \tilde{b}_{1} f_{1}\right)(x)\right| w(x) d x \\
& \leq C \sum_{\left|\alpha_{2}\right|=m_{2}}\left\|D^{\alpha_{2}} b_{2}\right\|_{B M O} \sum_{\left|\alpha_{1}\right|=m_{1}}\left(\frac{1}{w(Q)} \int_{R^{n}}\left|T\left(D^{\alpha_{1}} \tilde{b}_{1} f_{1}\right)(x)\right|^{p} w(x) d x\right)^{1 / p}
\end{aligned}
$$


Chang and Gro Journal of Inequalities and Applications 2014, 2014:341

Page 6 of 15

http://www.journalofinequalitiesandapplications.com/content/2014/1/341

$$
\begin{aligned}
\leq & C \sum_{\left|\alpha_{2}\right|=m_{2}}\left\|D^{\alpha_{2}} b_{2}\right\|_{B M O} \sum_{\left|\alpha_{1}\right|=m_{1}}\left(\frac{1}{w(Q)} \int_{R^{n}}\left|D^{\alpha_{1}} \tilde{b}_{1}(x) f_{1}(x)\right|^{p} w(x) d x\right)^{1 / p} \\
\leq & C \sum_{\left|\alpha_{2}\right|=m_{2}}\left\|D^{\alpha_{2}} b_{2}\right\|_{B M O} \sum_{\left|\alpha_{1}\right|=m_{1}}\left(\frac{1}{|Q|} \int_{\tilde{Q}}\left|D^{\alpha_{1}} b_{1}(x)-\left(D^{\alpha_{1}} b_{1}\right)_{\tilde{Q}}\right|^{p p_{0}^{\prime}} d x\right)^{1 / p p_{0}^{\prime}} \\
& \times w(Q)^{-1 / p}|Q|^{1 / p}\left(\frac{1}{|\tilde{Q}|} \int_{\tilde{Q}} w(x)^{p_{0}} d x\right)^{1 / p p_{0}}\|f\|_{L^{\infty}(w)} \\
\leq & C \prod_{j=1}^{2}\left(\sum_{\left|\alpha_{j}\right|=m_{j}}\left\|D^{\alpha_{j}} b_{j}\right\|_{B M O}\right) w(Q)^{-1 / p}|Q|^{1 / p}\left(\frac{1}{|\tilde{Q}|} \int_{\tilde{Q}} w(x) d x\right)^{1 / p}\|f\|_{L^{\infty}(w)} \\
\leq & C \prod_{j=1}^{2}\left(\sum_{\left|\alpha_{j}\right|=m_{j}}\left\|D^{\alpha_{j}} b_{j}\right\|_{B M O}\right)\|f\|_{L^{\infty}(w) .}
\end{aligned}
$$

For $I_{3}$, similar to the proof of $I_{2}$, we get

$$
I_{3} \leq C \prod_{j=1}^{2}\left(\sum_{\left|\alpha_{j}\right|=m_{j}}\left\|D^{\alpha_{j}} b_{j}\right\|_{B M O}\right)\|f\|_{L^{\infty}(w)} .
$$

Similarly, for $I_{4}$, choose $1<r_{1}, r_{2}<\infty$ such that $1 / r_{1}+1 / r_{2}+1 / p_{0}=1$, we obtain, by Hölder's inequality and the reverse of Hölder's inequality,

$$
\begin{aligned}
I_{4} \leq & C \sum_{\left|\alpha_{1}\right|=m_{1},\left|\alpha_{2}\right|=m_{2}} \frac{1}{w(Q)} \int_{Q}\left|T\left(D^{\alpha_{1}} \tilde{b}_{1} D^{\alpha_{2}} \tilde{b}_{2} f_{1}\right)(x)\right| w(x) d x \\
\leq & C \sum_{\left|\alpha_{1}\right|=m_{1},\left|\alpha_{2}\right|=m_{2}}\left(\frac{1}{w(Q)} \int_{R^{n}}\left|T\left(D^{\alpha_{1}} \tilde{b}_{1} D^{\alpha_{2}} \tilde{b}_{2} f_{1}\right)(x)\right|^{p} w(x) d x\right)^{1 / p} \\
\leq & C \sum_{\left|\alpha_{1}\right|=m_{1},\left|\alpha_{2}\right|=m_{2}} w(Q)^{-1 / p}\left(\int_{R^{n}}\left|D^{\alpha_{1}} \tilde{b}_{1}(x) D^{\alpha_{2}} \tilde{b}_{2}(x) f_{1}(x)\right|^{p} w(x) d x\right)^{1 / p} \\
\leq & C \sum_{\left|\alpha_{1}\right|=m_{1},\left|\alpha_{2}\right|=m_{2}}\left(\frac{1}{|\tilde{Q}|} \int_{\tilde{Q}}\left|D^{\alpha_{1}} \tilde{b}_{1}(x)\right|^{p r_{1}} d x\right)^{1 / p r_{1}}\left(\frac{1}{|\tilde{Q}|} \int_{\tilde{Q}}\left|D^{\alpha_{2}} \tilde{b}_{2}(x)\right|^{p r_{2}} d x\right)^{1 / p r_{2}} \\
& \times w(Q)^{-1 / p}|Q|^{1 / p}\left(\frac{1}{|\tilde{Q}|} \int_{\tilde{Q}} w(x)^{p_{0}} d x\right)^{1 / p p_{0}}\|f\|_{L^{\infty}(w)} \\
\leq & C \prod_{j=1}^{2}\left(\sum_{\left|\alpha_{j}\right|=m_{j}}\left\|D^{\alpha_{j}} b_{j}\right\|_{B M O}\right)\|f\|_{L^{\infty}(w) .}
\end{aligned}
$$

For $I_{5}$, we write

$$
\begin{aligned}
& T_{\tilde{b}}\left(f_{2}\right)(x)-T_{\tilde{b}}\left(f_{2}\right)\left(x_{0}\right) \\
& =\int_{R^{n}}\left(\frac{K(x, y)}{|x-y|^{m}}-\frac{K\left(x_{0}, y\right)}{\left|x_{0}-y\right|^{m}}\right) \prod_{j=1}^{2} R_{m_{j}}\left(\tilde{b}_{j} ; x, y\right) f_{2}(y) d y \\
& \quad+\int_{R^{n}}\left(R_{m_{1}}\left(\tilde{b}_{1} ; x, y\right)-R_{m_{1}}\left(\tilde{b}_{1} ; x_{0}, y\right)\right) \frac{R_{m_{2}}\left(\tilde{b}_{2} ; x, y\right)}{\left|x_{0}-y\right|^{m}} K\left(x_{0}, y\right) f_{2}(y) d y
\end{aligned}
$$




$$
\begin{aligned}
& +\int_{R^{n}}\left(R_{m_{2}}\left(\tilde{b}_{2} ; x, y\right)-R_{m_{2}}\left(\tilde{b}_{2} ; x_{0}, y\right)\right) \frac{R_{m_{1}}\left(\tilde{b}_{1} ; x_{0}, y\right)}{\left|x_{0}-y\right|^{m}} K\left(x_{0}, y\right) f_{2}(y) d y \\
& -\sum_{\left|\alpha_{1}\right|=m_{1}} \frac{1}{\alpha_{1} !} \int_{R^{n}}\left[\frac{R_{m_{2}}\left(\tilde{b}_{2} ; x, y\right)(x-y)^{\alpha_{1}}}{|x-y|^{m}} K(x, y)-\frac{R_{m_{2}}\left(\tilde{b}_{2} ; x_{0}, y\right)\left(x_{0}-y\right)^{\alpha_{1}}}{\left|x_{0}-y\right|^{m}} K\left(x_{0}, y\right)\right] \\
& \times D^{\alpha_{1}} \tilde{b}_{1}(y) f_{2}(y) d y \\
& -\sum_{\left|\alpha_{2}\right|=m_{2}} \frac{1}{\alpha_{2} !} \int_{R^{n}}\left[\frac{R_{m_{1}}\left(\tilde{b}_{1} ; x, y\right)(x-y)^{\alpha_{2}}}{|x-y|^{m}} K(x, y)-\frac{R_{m_{1}}\left(\tilde{b}_{1} ; x_{0}, y\right)\left(x_{0}-y\right)^{\alpha_{2}}}{\left|x_{0}-y\right|^{m}} K\left(x_{0}, y\right)\right] \\
& \times D^{\alpha_{2}} \tilde{b}_{2}(y) f_{2}(y) d y \\
& +\sum_{\left|\alpha_{1}\right|=m_{1},\left|\alpha_{2}\right|=m_{2}} \frac{1}{\alpha_{1} ! \alpha_{2} !} \int_{R^{n}}\left[\frac{(x-y)^{\alpha_{1}+\alpha_{2}}}{|x-y|^{m}} K(x, y)-\frac{\left(x_{0}-y\right)^{\alpha_{1}+\alpha_{2}}}{\left|x_{0}-y\right|^{m}} K\left(x_{0}, y\right)\right] \\
& \quad \times D^{\alpha_{1}} \tilde{b}_{1}(y) D^{\alpha_{2}} \tilde{b}_{2}(y) f_{2}(y) d y \\
& =I_{5}^{(1)}(x)+I_{5}^{(2)}(x)+I_{5}^{(3)}(x)+I_{5}^{(4)}(x)+I_{5}^{(5)}(x)+I_{5}^{(6)}(x) .
\end{aligned}
$$

By Lemma 1 and the inequality (see [9])

$$
\left|b_{Q_{1}}-b_{Q_{2}}\right| \leq C \log \left(\left|Q_{2}\right| /\left|Q_{1}\right|\right)\|b\|_{B M O} \quad \text { for } Q_{1} \subset Q_{2},
$$

we know that, for $x \in Q$ and $y \in 2^{k+1} \tilde{Q} \backslash 2^{k} \tilde{Q}$,

$$
\begin{aligned}
\left|R_{m_{j}}\left(\tilde{b}_{j} ; x, y\right)\right| & \leq C|x-y|^{m_{j}} \sum_{|\alpha|=m_{j}}\left(\left\|D^{\alpha} b_{j}\right\|_{B M O}+\left|\left(D^{\alpha} b_{j}\right)_{\tilde{Q}(x, y)}-\left(D^{\alpha} b_{j}\right)_{\tilde{Q}}\right|\right) \\
& \leq C k|x-y|^{m_{j}} \sum_{|\alpha|=m_{j}}\left\|D^{\alpha} b_{j}\right\|_{B M O} .
\end{aligned}
$$

Note that $|x-y| \sim\left|x_{0}-y\right|$ for $x \in Q$ and $y \in R^{n} \backslash \tilde{Q}$, we obtain, by the conditions on $K$,

$$
\begin{aligned}
\left|I_{5}^{(1)}(x)\right| \leq & \int_{R^{n}}\left|\frac{1}{|x-y|^{m}}-\frac{1}{\left|x_{0}-y\right|^{m}}\right||K(x, y)| \prod_{j=1}^{2}\left|R_{m_{j}}\left(\tilde{b}_{j} ; x, y\right)\right|\left|f_{2}(y)\right| d y \\
& +\int_{R^{n}}\left|K(x, y)-K\left(x_{0}, y\right)\right|\left|x_{0}-y\right|^{-m} \prod_{j=1}^{2}\left|R_{m_{j}}\left(\tilde{b}_{j} ; x, y\right)\right|\left|f_{2}(y)\right| d y \\
\leq & \sum_{k=0}^{\infty} \int_{2^{k+1} \tilde{Q} \backslash 2^{k} \tilde{Q} \mid}\left|\frac{1}{|x-y|^{m}}-\frac{1}{\left|x_{0}-y\right|^{m}}\right||K(x, y)| \prod_{j=1}^{2}\left|R_{m_{j}}\left(\tilde{b}_{j} ; x, y\right)\right||f(y)| d y \\
& +\sum_{k=0}^{\infty} \int_{2^{k+1} \tilde{Q} \backslash 2^{k} \tilde{Q}}\left|K(x, y)-K\left(x_{0}, y\right)\right|\left|x_{0}-y\right|^{-m} \prod_{j=1}^{2}\left|R_{m_{j}}\left(\tilde{b}_{j} ; x, y\right)\right||f(y)| d y \\
\leq & C \prod_{j=1}^{2}\left(\sum_{\left|\alpha_{j}\right|=m_{j}}\left\|D^{\alpha_{j}} b_{j}\right\|_{B M O}\right) \sum_{k=0}^{\infty} k^{2} \int_{2^{k+1} \tilde{Q} \backslash 2^{k} \tilde{Q}} \frac{\left|x-x_{0}\right|}{\left|x_{0}-y\right|^{n+1}}|f(y)| d y \\
& +C \prod_{j=1}^{2}\left(\sum_{\left|\alpha_{j}\right|=m_{j}}\left\|D^{\alpha_{j}} b_{j}\right\|_{B M O}\right) \sum_{k=0}^{\infty} k^{2}\left(\int_{2^{k+1} \tilde{Q} \backslash 2^{k} \tilde{Q}}|f(y)|^{q^{\prime}} d y\right)^{1 / q^{\prime}}
\end{aligned}
$$




$$
\begin{aligned}
& \times\left(\int_{2^{k+1} \tilde{Q} \backslash 2^{k} \tilde{Q}}\left|K(x, y)-K\left(x_{0}, y\right)\right|^{q} d y\right)^{1 / q} \\
\leq & C \prod_{j=1}^{2}\left(\sum_{\left|\alpha_{j}\right|=m_{j}}\left\|D^{\alpha_{j}} b_{j}\right\|_{B M O}\right) \sum_{k=1}^{\infty} k^{2}\left(2^{-k}+C_{k}\right)\|f\|_{L^{\infty}(w)} \\
\leq & C \prod_{j=1}^{2}\left(\sum_{\left|\alpha_{j}\right|=m_{j}}\left\|D^{\alpha_{j}} b_{j}\right\|_{B M O}\right)\|f\|_{L^{\infty}(w) .}
\end{aligned}
$$

For $I_{5}^{(2)}(x)$, by the formula (see [3]):

$$
R_{m_{j}}\left(\tilde{b}_{j} ; x, y\right)-R_{m_{j}}\left(\tilde{b}_{j} ; x_{0}, y\right)=\sum_{|\beta|<m} \frac{1}{\beta !} R_{m-|\beta|}\left(D^{\beta} \tilde{b}_{j} ; x, x_{0}\right)(x-y)^{\beta}
$$

and Lemma 1, we have

$$
\left|R_{m_{j}}\left(\tilde{b}_{j} ; x, y\right)-R_{m_{j}}\left(\tilde{b}_{j} ; x_{0}, y\right)\right| \leq C \sum_{|\beta|<m_{j}} \sum_{|\alpha|=m_{j}}\left|x-x_{0}\right|^{m_{j}-|\beta|}|x-y|^{|\beta|}\left\|D^{\alpha} b_{j}\right\|_{B M O^{\prime}}
$$

thus

$$
\begin{aligned}
\left|I_{5}^{(2)}(x)\right| & \leq C \prod_{j=1}^{2}\left(\sum_{\left|\alpha_{j}\right|=m_{j}}\left\|D^{\alpha_{j}} b_{j}\right\|_{B M O}\right) \sum_{k=0}^{\infty} \int_{2^{k+1} \tilde{Q} \mid 2^{k} \tilde{Q}} k \frac{\left|x-x_{0}\right|}{\left|x_{0}-y\right|^{n+1}}|f(y)| d y \\
& \leq C \prod_{j=1}^{2}\left(\sum_{\left|\alpha_{j}\right|=m_{j}}\left\|D^{\alpha_{j}} b_{j}\right\|_{B M O}\right) \sum_{k=1}^{\infty} k 2^{-k}\|f\|_{L^{\infty}(w)} \\
& \leq C \prod_{j=1}^{2}\left(\sum_{\left|\alpha_{j}\right|=m_{j}}\left\|D^{\alpha_{j}} b_{j}\right\|_{B M O}\right)\|f\|_{L^{\infty}(w)} .
\end{aligned}
$$

Similarly,

$$
\left|I_{5}^{(3)}(x)\right| \leq C \prod_{j=1}^{2}\left(\sum_{\left|\alpha_{j}\right|=m_{j}}\left\|D^{\alpha_{j}} b_{j}\right\|_{B M O}\right)\|f\|_{L^{\infty}(w)} .
$$

For $I_{5}^{(4)}(x)$, similar to the proof of $I_{5}^{(1)}(x)$ and $I_{5}^{(2)}(x)$, we get

$$
\begin{aligned}
\left|I_{5}^{(4)}(x)\right| \leq & C \sum_{\left|\alpha_{1}\right|=m_{1}} \int_{R^{n}}\left|R_{m_{2}}\left(\tilde{b}_{2} ; x, y\right)-R_{m_{2}}\left(\tilde{b}_{2} ; x_{0}, y\right)\right| \frac{\left|\left(x_{0}-y\right)^{\alpha_{1}} K\left(x_{0}, y\right)\right|}{\left|x_{0}-y\right|^{m}} \\
& \times\left|D^{\alpha_{1}} \tilde{b}_{1}(y)\right|\left|f_{2}(y)\right| d y \\
& +C \sum_{\left|\alpha_{1}\right|=m_{1}} \int_{R^{n}}\left|\frac{(x-y)^{\alpha_{1}}}{|x-y|^{m}}-\frac{\left(x_{0}-y\right)^{\alpha_{1}}}{\left|x_{0}-y\right|^{m}}\right||K(x, y)|\left|R_{m_{2}}\left(\tilde{b}_{2} ; x, y\right)\right| \\
& \times\left|D^{\alpha_{1}} \tilde{b}_{1}(y)\right|\left|f_{2}(y)\right| d y \\
& +C \sum_{\left|\alpha_{1}\right|=m_{1}} \int_{R^{n}}\left|K(x, y)-K\left(x_{0}, y\right)\right|\left|\frac{\left.\mid x_{0}-y\right)^{\alpha_{1}}}{\left|x_{0}-y\right|^{m}}\right|\left|R_{m_{2}}\left(\tilde{b}_{2} ; x, y\right)\right|
\end{aligned}
$$




$$
\begin{aligned}
& \times\left|D^{\alpha_{1}} \tilde{b}_{1}(y)\right|\left|f_{2}(y)\right| d y \\
\leq & C \sum_{\left|\alpha_{2}\right|=m_{2}}\left\|D^{\alpha_{2}} b_{2}\right\|_{B M O} \sum_{k=1}^{\infty} k 2^{-k} \sum_{\left|\alpha_{1}\right|=m_{1}}\left(\frac{1}{\left|2^{k} \tilde{Q}\right|} \int_{2^{k} \tilde{Q}}\left|D^{\alpha_{1}} \tilde{b}_{1}(y)\right| d y\right)\|f\|_{L^{\infty}(w)} \\
& +C \sum_{\left|\alpha_{2}\right|=m_{2}}\left\|D^{\alpha_{2}} b_{2}\right\|_{B M O} \sum_{\left|\alpha_{1}\right|=m_{1}} \sum_{k=0}^{\infty} k\left(\int_{2^{k+1} \tilde{Q} 2^{k} \tilde{Q}}\left|K(x, y)-K\left(x_{0}, y\right)\right|^{q} d y\right)^{1 / q} \\
& \times\left(\int_{2^{k+1} \tilde{Q} \mid 2^{k} \tilde{Q}}\left|D^{\alpha_{1}} \tilde{b}_{1}(y)\right|^{q^{\prime}} d y\right)^{1 / q^{\prime}}\|f\|_{L^{\infty}(w)} \\
\leq & C \prod_{j=1}^{2}\left(\sum_{\left|\alpha_{j}\right|=m_{j}}\left\|D^{\alpha_{j}} b_{j}\right\|_{B M O}\right) \sum_{k=1}^{\infty} k^{2}\left(2^{-k}+C_{k}\right)\|f\|_{L^{\infty}(w)} \\
\leq & C \prod_{j=1}^{2}\left(\sum_{\left|\alpha_{j}\right|=m_{j}}\left\|D^{\alpha_{j}} b_{j}\right\|_{B M O}\right)\|f\|_{L^{\infty}(w) .}
\end{aligned}
$$

Similarly,

$$
\left|I_{5}^{(5)}(x)\right| \leq C \prod_{j=1}^{2}\left(\sum_{\left|\alpha_{j}\right|=m_{j}}\left\|D^{\alpha_{j}} b_{j}\right\|_{B M O}\right)\|f\|_{L^{\infty}(w)} .
$$

For $I_{5}^{(6)}(x)$, taking $1<r_{1}, r_{2}<\infty$ such that $1 / r_{1}+1 / r_{2}=1$, then

$$
\begin{aligned}
\left|I_{5}^{(6)}(x)\right| \leq & C \sum_{\left|\alpha_{1}\right|=m_{1},\left|\alpha_{2}\right|=m_{2}} \int_{R^{n}}\left|\frac{(x-y)^{\alpha_{1}+\alpha_{2}} K(x, y)}{|x-y|^{m}}-\frac{\left(x_{0}-y\right)^{\alpha_{1}+\alpha_{2}} K\left(x_{0}, y\right)}{\left|x_{0}-y\right|^{m}}\right| \\
& \times\left|D^{\alpha_{1}} \tilde{b}_{1}(y)\right|\left|D^{\alpha_{2}} \tilde{b}_{2}(y)\right|\left|f_{2}(y)\right| d y \\
\leq & C \sum_{\left|\alpha_{1}\right|=m_{1},\left|\alpha_{2}\right|=m_{2}} \sum_{k=1}^{\infty}\left(2^{-k}+C_{k}\right)\|f\|_{L^{\infty}(w)} \\
& \times\left(\frac{1}{\left|2^{k} \tilde{Q}\right|} \int_{2^{k} \tilde{Q}}\left|D^{\alpha_{1}} \tilde{b}_{1}(y)\right|^{r_{1}} d y\right)^{1 / r_{1}}\left(\frac{1}{\left|2^{k} \tilde{Q}\right|} \int_{2^{k} \tilde{Q}}\left|D^{\alpha_{2}} \tilde{b}_{2}(y)\right|^{r_{2}} d y\right)^{1 / r_{2}} \\
\leq & C \prod_{j=1}^{2}\left(\sum_{\left|\alpha_{j}\right|=m_{j}}\left\|D^{\alpha_{j}} b_{j}\right\|_{B M O}\right) \sum_{k=1}^{\infty} k^{2}\left(2^{-k}+C_{k}\right)\|f\|_{L^{\infty}(w)} \\
\leq & C \prod_{j=1}^{2}\left(\sum_{\left|\alpha_{j}\right|=m_{j}}\left\|D^{\alpha_{j}} b_{j}\right\|_{B M O}\right)\|f\|_{L^{\infty}(w) .}
\end{aligned}
$$

Thus

$$
I_{5} \leq C \prod_{j=1}^{2}\left(\sum_{\left|\alpha_{j}\right|=m_{j}}\left\|D^{\alpha_{j}} b_{j}\right\|_{B M O}\right)\|f\|_{L^{\infty}(w) .}
$$

This completes the proof of Theorem 1 . 
Proof of Theorem 2 It is only for us to prove that there exists a constant $C_{Q}$ such that

$$
\frac{1}{w(Q)} \int_{Q}\left|T_{b}(f)(x)-C_{Q}\right| w(x) d x \leq C\|f\|_{B_{p}(w)}
$$

holds for any cube $Q=Q(0, d)$ with $d>1$. Without loss of generality, we may assume $l=2$. Fix a cube $Q=Q(0, d)$ with $d>1$. Let $\tilde{Q}=5 \sqrt{n} Q$ and $\tilde{b}_{j}(x)=b_{j}(x)-\sum_{|\alpha|=m_{j}} \frac{1}{\alpha !}\left(D^{\alpha} b_{j}\right)_{\tilde{Q}} x^{\alpha}$, then $R_{m_{j}}\left(b_{j} ; x, y\right)=R_{m_{j}}\left(\tilde{b}_{j} ; x, y\right)$ and $D^{\alpha} \tilde{b}_{j}=D^{\alpha} b_{j}-\left(D^{\alpha} b_{j}\right)_{\tilde{Q}}$ for $|\alpha|=m_{j}$. Similar to the proof of Theorem 1, we write, for $f_{1}=f \chi_{\tilde{Q}}$ and $f_{2}=f \chi_{R^{n} \backslash \tilde{Q}}$,

$$
\begin{aligned}
& \frac{1}{w(Q)} \int_{Q}\left|T_{b}(f)(x)-T_{\tilde{b}}\left(f_{2}\right)(0)\right| w(x) d x \\
& \leq \frac{1}{w(Q)} \int_{Q}\left|T\left(\frac{\prod_{j=1}^{2} R_{m_{j}}\left(\tilde{b}_{j} ; x, \cdot\right)}{|x-\cdot|^{m}} f_{1}\right)\right| w(x) d x \\
& \quad+\frac{1}{w(Q)} \int_{Q}\left|T\left(\sum_{\left|\alpha_{1}\right|=m_{1}} \frac{1}{\alpha_{1} !} \frac{R_{m_{2}}\left(\tilde{b}_{2} ; x, \cdot\right)(x-\cdot)^{\alpha_{1}} D^{\alpha_{1}} \tilde{b}_{1}}{|x-\cdot|^{m}} f_{1}\right)\right| w(x) d x \\
& \quad+\frac{1}{w(Q)} \int_{Q}\left|T\left(\sum_{\left|\alpha_{2}\right|=m_{2}} \frac{1}{\alpha_{2} !} \frac{R_{m_{1}}\left(\tilde{b}_{1} ; x, \cdot\right)(x-\cdot)^{\alpha_{2}} D^{\alpha_{2}} \tilde{b}_{2}}{|x-\cdot|^{m}} f_{1}\right)\right| w(x) d x \\
& \quad+\frac{1}{w(Q)} \int_{Q}\left|T\left(\sum_{\left|\alpha_{1}\right|=m_{1},\left|\alpha_{2}\right|=m_{2}} \frac{1}{\alpha_{1} ! \alpha_{2} !} \frac{(x-\cdot)^{\alpha_{1}+\alpha_{2}} D^{\alpha_{1}} \tilde{b}_{1} D^{\alpha_{2}} \tilde{b}_{2}}{|x-\cdot|^{m}} f_{1}\right)\right| w(x) d x \\
& \quad+\frac{1}{w(Q)} \int_{Q}\left|T_{\tilde{b}}\left(f_{2}\right)(x)-T_{\tilde{b}}\left(f_{2}\right)(0)\right| w(x) d x \\
& =L_{1}+L_{2}+L_{3}+L_{4}+L_{5} .
\end{aligned}
$$

Similar to the proof of Theorem 1, we get

$$
\begin{aligned}
L_{1} & \leq C \prod_{j=1}^{2}\left(\sum_{\left|\alpha_{j}\right|=m_{j}}\left\|D^{\alpha_{j}} b_{j}\right\|_{B M O}\right)\left(\frac{1}{w(Q)} \int_{R^{n}}\left|T\left(f_{1}\right)(x)\right|^{p} w(x) d x\right)^{1 / p} \\
& \leq C \prod_{j=1}^{2}\left(\sum_{\left|\alpha_{j}\right|=m_{j}}\left\|D^{\alpha_{j}} b_{j}\right\|_{B M O}\right) w(\tilde{Q})^{-1 / p}\left\|f \chi_{\tilde{Q}}\right\|_{L^{p}(w)} \\
& \leq C \prod_{j=1}^{2}\left(\sum_{\left|\alpha_{j}\right|=m_{j}}\left\|D^{\alpha_{j}} b_{j}\right\|_{B M O}\right)\|f\|_{B_{p}(w) .}
\end{aligned}
$$

For $L_{2}$, taking $r, s, t>1$ such that $r<p, t=p p_{0} /(p-r)$, and $1 / s+1 /(p / r)+1 / t=1$, then, by the reverse of Hölder's inequality,

$$
\begin{aligned}
L_{2} & \leq C \sum_{\left|\alpha_{2}\right|=m_{2}}\left\|D^{\alpha_{2}} b_{2}\right\|_{B M O} \sum_{\left|\alpha_{1}\right|=m_{1}}\left(\frac{1}{w(Q)} \int_{R^{n}}\left|T\left(D^{\alpha_{1}} \tilde{b}_{1} f_{1}\right)(x)\right|^{r} w(x) d x\right)^{1 / r} \\
& \leq C \sum_{\left|\alpha_{2}\right|=m_{2}}\left\|D^{\alpha_{2}} b_{2}\right\|_{B M O} w(Q)^{-1 / r} \sum_{\left|\alpha_{1}\right|=m_{1}}\left(\int_{R^{n}}\left|D^{\alpha_{1}} \tilde{b}_{1}(x) f_{1}(x)\right|^{r} w(x) d x\right)^{1 / r}
\end{aligned}
$$




$$
\begin{aligned}
\leq & C \sum_{\left|\alpha_{2}\right|=m_{2}}\left\|D^{\alpha_{2}} b_{2}\right\|_{B M O} w(Q)^{-1 / r} \sum_{\left|\alpha_{1}\right|=m_{1}}\left(\int_{\tilde{Q}}\left|D^{\alpha} \tilde{b}_{1}(x)\right|^{r s} d x\right)^{1 / r s} \\
& \times\left(\int_{\tilde{Q}}|f(x)|^{p} w(x) d x\right)^{1 / p}\left(\int_{\tilde{Q}} w(x)^{(1-r / p) t} d x\right)^{1 / r t} \\
\leq & C \prod_{j=1}^{2}\left(\sum_{\left|\alpha_{j}\right|=m_{j}}\left\|D^{\alpha_{j}} b_{j}\right\|_{B M O}\right) w(Q)^{-1 / r}|Q|^{1 / r s}\left\|f \chi_{\tilde{Q}}\right\|_{L^{p}(w)}|Q|^{1 / r t} \\
& \times\left(\frac{1}{|\tilde{Q}|} \int_{\tilde{Q}} w(x)^{p_{0}} d x\right)^{1 / r t} \\
\leq & C \prod_{j=1}^{2}\left(\sum_{\left|\alpha_{j}\right|=m_{j}}\left\|D^{\alpha_{j}} b_{j}\right\|_{B M O}\right) w(Q)^{-1 / r}|Q|^{1 / r s}\left\|f \chi_{\tilde{Q}}\right\|_{L^{p}(w)}\left(\frac{1}{|\tilde{Q}|} \int_{\tilde{Q}} w(x) d x\right)^{p_{0} / r t} \\
\leq & C \prod_{j=1}^{2}\left(\sum_{\left|\alpha_{j}\right|=m_{j}}\left\|D^{\alpha_{j}} b_{j}\right\|_{B M O}\right) w(\tilde{Q})^{-1 / p}\left\|f \chi_{\tilde{Q}}\right\|_{L^{p}(w)} \\
\leq & C \prod_{j=1}^{2}\left(\sum_{\left|\alpha_{j}\right|=m_{j}}\left\|D^{\alpha_{j}} b_{j}\right\|_{B M O}\right)\|f\|_{B_{p}(w)}, \\
L_{3} \leq & C \prod_{j=1}^{2}\left(\sum_{\left|\alpha_{j}\right|=m_{j}}\left\|D^{\alpha_{j}} b_{j}\right\|_{B M O}\right)\|f\|_{B_{p}(w) .}
\end{aligned}
$$

For $L_{4}$, taking $r, s_{1}, s_{2}, t>1$ such that $r<p, t=p p_{0} /(p-r)$, and $1 / s_{1}+1 / s_{2}+1 /(p / r)+1 / t=1$, then, by the reverse of Hölder's inequality,

$$
\begin{aligned}
L_{4} \leq & C \sum_{\left|\alpha_{1}\right|=m_{1},\left|\alpha_{2}\right|=m_{2}}\left(\frac{1}{w(Q)} \int_{R^{n}}\left|T\left(D^{\alpha_{1}} \tilde{b}_{1} D^{\alpha_{2}} \tilde{b}_{2} f_{1}\right)(x)\right|^{r} w(x) d x\right)^{1 / r} \\
\leq & C w(Q)^{-1 / r} \sum_{\left|\alpha_{1}\right|=m_{1},\left|\alpha_{2}\right|=m_{2}}\left(\int_{R^{n}}\left|D^{\alpha_{1}} \tilde{b}_{1}(x) D^{\alpha_{2}} \tilde{b}_{2}(x) f_{1}(x)\right|^{r} w(x) d x\right)^{1 / r} \\
\leq & C w(Q)^{-1 / r} \sum_{\left|\alpha_{1}\right|=m_{1},\left|\alpha_{2}\right|=m_{2}}\left(\int_{\tilde{Q}}\left|D^{\alpha} \tilde{b}_{1}(x)\right|^{r s_{1}} d x\right)^{1 / r_{1}}\left(\int_{\tilde{Q}}\left|D^{\alpha} \tilde{b}_{2}(x)\right|^{r s_{2}} d x\right)^{1 / r s_{2}} \\
& \times\left(\int_{\tilde{Q}}|f(x)|^{p} w(x) d x\right)^{1 / p}\left(\int_{\tilde{Q}} w(x)^{(1-r / p) t} d x\right)^{1 / r t} \\
\leq & C \prod_{j=1}^{2}\left(\sum_{\left|\alpha_{j}\right|=m_{j}}\left\|D^{\alpha_{j}} b_{j}\right\|_{B M O}\right) w(Q)^{-1 / r}|Q|^{1 / r r_{1}+1 / r s_{2}+1 / r t}\left\|f \chi_{\tilde{Q}}\right\|_{L^{p}(w)} \\
& \times\left(\frac{1}{|\tilde{Q}|} \int_{\tilde{Q}} w(x) d x\right)^{p_{0} / r t} \\
\leq & C \prod_{j=1}^{2}\left(\sum_{\left|\alpha_{j}\right|=m_{j}}\left\|D^{\alpha_{j}} b_{j}\right\|_{B M O}\right) w(\tilde{Q})^{-1 / p}\left\|f \chi_{\tilde{Q}}\right\|_{L^{p}(w)} \\
\leq & C \prod_{j=1}^{2}\left(\sum_{\left|\alpha_{j}\right|=m_{j}}\left\|D^{\alpha_{j}} b_{j}\right\|_{B M O}\right)\|f\|_{B_{p}(w) .}
\end{aligned}
$$


For $L_{5}$, similar to the proof of the proof of $I_{5}$ in Theorem 1 , we have

$$
\begin{aligned}
& T_{\tilde{b}}\left(f_{2}\right)(x)-T_{\tilde{b}}\left(f_{2}\right)(0) \\
& =\int_{R^{n}}\left(\frac{K(x, y)}{|x-y|^{m}}-\frac{K(0, y)}{|y|^{m}}\right) \prod_{j=1}^{2} R_{m_{j}}\left(\tilde{b}_{j} ; x, y\right) f_{2}(y) d y \\
& +\int_{R^{n}}\left(R_{m_{1}}\left(\tilde{b}_{1} ; x, y\right)-R_{m_{1}}\left(\tilde{b}_{1} ; 0, y\right)\right) \frac{R_{m_{2}}\left(\tilde{b}_{2} ; x, y\right)}{|y|^{m}} K(0, y) f_{2}(y) d y \\
& +\int_{R^{n}}\left(R_{m_{2}}\left(\tilde{b}_{2} ; x, y\right)-R_{m_{2}}\left(\tilde{b}_{2} ; 0, y\right)\right) \frac{R_{m_{1}}\left(\tilde{b}_{1} ; x_{0}, y\right)}{|y|^{m}} K(0, y) f_{2}(y) d y \\
& -\sum_{\left|\alpha_{1}\right|=m_{1}} \frac{1}{\alpha_{1} !} \int_{R^{n}}\left[\frac{R_{m_{2}}\left(\tilde{b}_{2} ; x, y\right)(x-y)^{\alpha_{1}}}{|x-y|^{m}} K(x, y)-\frac{R_{m_{2}}\left(\tilde{b}_{2} ; 0, y\right)(-y)^{\alpha_{1}}}{|y|^{m}} K(0, y)\right] \\
& \times D^{\alpha_{1}} \tilde{b}_{1}(y) f_{2}(y) d y \\
& -\sum_{\left|\alpha_{2}\right|=m_{2}} \frac{1}{\alpha_{2} !} \int_{R^{n}}\left[\frac{R_{m_{1}}\left(\tilde{b}_{1} ; x, y\right)(x-y)^{\alpha_{2}}}{|x-y|^{m}} K(x, y)-\frac{R_{m_{1}}\left(\tilde{b}_{1} ; 0, y\right)(-y)^{\alpha_{2}}}{|y|^{m}} K(0, y)\right] \\
& \times D^{\alpha_{2}} \tilde{b}_{2}(y) f_{2}(y) d y \\
& +\sum_{\left|\alpha_{1}\right|=m_{1},\left|\alpha_{2}\right|=m_{2}} \frac{1}{\alpha_{1} ! \alpha_{2} !} \int_{R^{n}}\left[\frac{(x-y)^{\alpha_{1}+\alpha_{2}}}{|x-y|^{m}} K(x, y)-\frac{(-y)^{\alpha_{1}+\alpha_{2}}}{|y|^{m}} K(0, y)\right] \\
& \times D^{\alpha_{1}} \tilde{b}_{1}(y) D^{\alpha_{2}} \tilde{b}_{2}(y) f_{2}(y) d y \\
& =L_{5}^{(1)}(x)+L_{5}^{(2)}(x)+L_{5}^{(3)}(x)+L_{5}^{(4)}(x)+L_{5}^{(5)}(x)+L_{5}^{(6)}(x) .
\end{aligned}
$$

For $L_{5}^{(1)}(x)$, taking $1<r<\infty$ such that $1 / p+1 / q+1 / r=1$, by $w \in A_{1} \subset A_{p / r+1}$, we get

$$
\begin{aligned}
\left|L_{5}^{(1)}(x)\right| \leq & C \sum_{k=0}^{\infty} \int_{2^{k+1} \tilde{Q} \backslash 2^{k} \tilde{Q}}\left|\frac{1}{|x-y|^{m}}-\frac{1}{|y|^{m}}\right||K(x, y)| \prod_{j=1}^{2}\left|R_{m_{j}}\left(\tilde{b}_{j} ; x, y\right)\right||f(y)| d y \\
& +\sum_{k=0}^{\infty} \int_{2^{k+1} \tilde{Q} \backslash 2^{k} \tilde{Q}}|K(x, y)-K(0, y)||y|^{-m} \\
& \times \prod_{j=1}^{2}\left|R_{m_{j}}\left(\tilde{b}_{j} ; x, y\right)\right| f(y) \mid w(y)^{1 / p} w(y)^{-1 / p} d y \\
\leq & C \prod_{j=1}^{2}\left(\sum_{|\alpha|=m_{j}}\left\|D^{\alpha} b_{j}\right\|_{B M O}\right) \sum_{k=0}^{\infty} \int_{2^{k+1} \tilde{Q} \backslash 2^{k} \tilde{Q}} k^{2} \frac{d}{\left(2^{k} d\right)^{n+1}}|f(y)| d y \\
& +C \prod_{j=1}^{2}\left(\sum_{|\alpha|=m_{j}}\left\|D^{\alpha} b_{j}\right\|_{B M O}\right) \sum_{k=0}^{\infty} k^{2}\left(\int_{2^{k+1} \tilde{Q} \backslash 2^{k} \tilde{Q}}|K(x, y)-K(0, y)|^{q} d y\right)^{1 / q} \\
& \times\left(\int_{2^{k+1} \tilde{Q}}|f(y)|^{p} w(y) d y\right)^{1 / p}\left(\int_{2^{k+1} \tilde{Q}} w(y)^{-r / p} d y\right)^{1 / r} \\
\leq & C \prod_{j=1}^{2}\left(\sum_{\left|\alpha_{j}\right|=m_{j}}\left\|D^{\alpha_{j}} b_{j}\right\|_{B M O}\right) \sum_{k=1}^{\infty} k^{2} 2^{-k} w\left(2^{k} \tilde{Q}\right)^{-1 / p}\left(\int_{2^{k} \tilde{Q}}|f(y)|^{p} w(y) d y\right)^{1 / p}
\end{aligned}
$$


Chang and Gu Journal of Inequalities and Applications 2014, 2014:341

Page 13 of 15

http://www.journalofinequalitiesandapplications.com/content/2014/1/341

$$
\begin{aligned}
& \times\left(\frac{1}{\left|2^{k} \tilde{Q}\right|} \int_{2^{k} \tilde{Q}} w(y) d y\right)^{1 / p}\left(\frac{1}{\left|2^{k} \tilde{Q}\right|} \int_{2^{k} \tilde{Q}} w(y)^{-1 /(p-1)} d y\right)^{(p-1) / p} \\
& +C \prod_{j=1}^{2}\left(\sum_{\left|\alpha_{j}\right|=m_{j}}\left\|D^{\alpha_{j}} b_{j}\right\|_{B M O}\right) \sum_{k=1}^{\infty} k^{2} C_{k} w\left(2^{k} \tilde{Q}\right)^{-1 / p}\left(\int_{2^{k} \tilde{Q}}|f(y)|^{p} w(y) d y\right)^{1 / p} \\
& \times\left(\frac{1}{\left|2^{k} \tilde{Q}\right|} \int_{2^{k} \tilde{Q}} w(y) d y\right)^{1 / p}\left(\frac{1}{\left|2^{k} \tilde{Q}\right|} \int_{2^{k} \tilde{Q}} w(y)^{-r / p} d y\right)^{1 / r} \\
\leq & C \prod_{j=1}^{2}\left(\sum_{\left|\alpha_{j}\right|=m_{j}}\left\|D^{\alpha_{j}} b_{j}\right\|_{B M O}\right)\|f\|_{B_{p}(w)} .
\end{aligned}
$$

Similarly, we get, for $1<r_{1}, r_{2}, r_{3}, r_{4}, s<\infty$ with $1 / p+1 / r_{1}+1 / s=1,1 / p+1 / q+1 / r_{2}+1 / s=1$, and $1 / p+1 / q+1 / r_{3}+1 / r_{4}+1 / s=1$,

$$
\begin{aligned}
& \left|L_{5}^{(2)}(x)+L_{5}^{(3)}(x)+L_{5}^{(4)}(x)+L_{5}^{(5)}(x)+L_{5}^{(6)}(x)\right| \\
& \leq C\left(\sum_{|\alpha|=m_{2}}\left\|D^{\alpha} b_{2}\right\|_{B M O}\right) \sum_{|\alpha|=m_{1}} \sum_{k=0}^{\infty} \int_{2^{k+1} \tilde{Q} \backslash 2^{k} \tilde{Q}} k \frac{d}{\left(2^{k} d\right)^{n+1}}\left|D^{\alpha_{1}} \tilde{b}_{1}(y)\right||f(y)| d y \\
& +C\left(\sum_{|\alpha|=m_{1}}\left\|D^{\alpha} b_{1}\right\|_{B M O}\right) \sum_{|\alpha|=m_{2}} \sum_{k=0}^{\infty} \int_{2^{k+1} \tilde{Q} \backslash 2^{k} \tilde{Q}} k \frac{d}{\left(2^{k} d\right)^{n+1}}\left|D^{\alpha_{2}} \tilde{b}_{2}(y)\right||f(y)| d y \\
& +C\left(\sum_{|\alpha|=m_{2}}\left\|D^{\alpha} b_{2}\right\|_{B M O}\right) \sum_{|\alpha|=m_{1}} \sum_{k=0}^{\infty} k \int_{2^{k+1} \tilde{Q} \backslash 2^{k} \tilde{Q}}|K(x, y)-K(0, y)| \\
& \times\left|D^{\alpha_{1}} \tilde{b}_{1}(y)\right||f(y)| d y \\
& +C\left(\sum_{|\alpha|=m_{1}}\left\|D^{\alpha} b_{1}\right\|_{B M O}\right) \sum_{|\alpha|=m_{2}} \sum_{k=0}^{\infty} k \int_{2^{k+1} \tilde{Q} \backslash 2^{k} \tilde{Q}}|K(x, y)-K(0, y)| \\
& \times\left|D^{\alpha_{2}} \tilde{b}_{2}(y)\right||f(y)| d y \\
& +C \sum_{\left|\alpha_{1}\right|=m_{1},\left|\alpha_{2}\right|=m_{2}} \sum_{k=0}^{\infty} \int_{2^{k+1} \tilde{Q} \backslash 2^{k} \tilde{Q}}|K(x, y)-K(0, y)|\left|D^{\alpha_{1}} \tilde{b}_{1}(y)\right|\left|D^{\alpha_{2}} \tilde{b}_{2}(y)\right||f(y)| d y \\
& \leq C\left(\sum_{|\alpha|=m_{2}}\left\|D^{\alpha} b_{2}\right\|_{B M O}\right) \sum_{|\alpha|=m_{1}} \sum_{k=0}^{\infty} k\left(\int_{2^{k+1} \tilde{Q}}\left|D^{\alpha_{1}} \tilde{b}_{1}(y)\right|^{r_{1}} d y\right)^{1 / r_{1}} \\
& \times\left(\int_{2^{k+1} \tilde{Q}}|f(y)|^{p} w(y) d y\right)^{1 / p}\left(\int_{2^{k+1} \tilde{Q}} w(y)^{-s / p} d y\right)^{1 / s} \\
& +C\left(\sum_{|\alpha|=m_{1}}\left\|D^{\alpha} b_{1}\right\|_{B M O}\right) \sum_{|\alpha|=m_{2}} \sum_{k=0}^{\infty} k\left(\int_{2^{k+1} \tilde{Q}}\left|D^{\alpha_{2}} \tilde{b}_{2}(y)\right|^{r_{1}} d y\right)^{1 / r_{1}} \\
& \times\left(\int_{2^{k+1} \tilde{Q}}|f(y)|^{p} w(y) d y\right)^{1 / p}\left(\int_{2^{k+1} \tilde{Q}} w(y)^{-s / p} d y\right)^{1 / s} \\
& +C\left(\sum_{|\alpha|=m_{2}}\left\|D^{\alpha} b_{2}\right\|_{B M O}\right) \sum_{|\alpha|=m_{1}} \sum_{k=0}^{\infty} k\left(\int_{2^{k+1} \tilde{Q} \backslash 2^{k} \tilde{Q}}|K(x, y)-K(0, y)|^{q} d y\right)^{1 / q} \\
& \times\left(\int_{2^{k+1} \tilde{Q}}\left|D^{\alpha_{1}} \tilde{b}_{1}(y)\right|^{r_{2}} d y\right)^{1 / r_{2}}\left(\int_{2^{k+1} \tilde{Q}}|f(y)|^{p} w(y) d y\right)^{1 / p}\left(\int_{2^{k+1} \tilde{Q}} w(y)^{-s / p} d y\right)^{1 / s}
\end{aligned}
$$




$$
\begin{aligned}
& +C\left(\sum_{|\alpha|=m_{1}}\left\|D^{\alpha} b_{1}\right\|_{B M O}\right) \sum_{|\alpha|=m_{2}} \sum_{k=0}^{\infty} k\left(\int_{2^{k+1} \tilde{Q} \backslash 2^{k} \tilde{Q}}|K(x, y)-K(0, y)|^{q} d y\right)^{1 / q} \\
& \times\left(\int_{2^{k+1} \tilde{Q}}\left|D^{\alpha_{2}} \tilde{b}_{2}(y)\right|^{r_{2}} d y\right)^{1 / r_{2}}\left(\int_{2^{k+1} \tilde{Q}}|f(y)|^{p} w(y) d y\right)^{1 / p}\left(\int_{2^{k+1} \tilde{Q}} w(y)^{-s / p} d y\right)^{1 / s} \\
& +C \sum_{\left|\alpha_{1}\right|=m_{1},\left|\alpha_{2}\right|=m_{2}} \sum_{k=0}^{\infty}\left(\int_{2^{k+1} \tilde{Q} \backslash 2^{k} \tilde{Q}}|K(x, y)-K(0, y)|^{q} d y\right)^{1 / q}\left(\int_{2^{k+1} \tilde{Q}} w(y)^{-s / p} d y\right)^{1 / s} \\
& \times\left(\int_{2^{k+1} \tilde{Q}}\left|D^{\alpha_{1}} \tilde{b}_{1}(y)\right|^{r_{3}} d y\right)^{1 / r_{3}}\left(\int_{2^{k+1} \tilde{Q}}\left|D^{\alpha_{2}} \tilde{b}_{2}(y)\right|^{r_{4}} d y\right)^{1 / r_{4}} \\
& \times\left(\int_{2^{k+1} \tilde{Q}}|f(y)|^{p} w(y) d y\right)^{1 / p} \\
& \leq C \prod_{j=1}^{2}\left(\sum_{\left|\alpha_{j}\right|=m_{j}}\left\|D^{\alpha_{j}} b_{j}\right\|_{B M O}\right) \sum_{k=1}^{\infty} k^{2}\left(2^{-k}+C_{k}\right) w\left(2^{k} \tilde{Q}\right)^{-1 / p}\left(\int_{2^{k} \tilde{Q}}|f(y)|^{p} w(y) d y\right)^{1 / p} \\
& \leq C \prod_{j=1}^{2}\left(\sum_{\left|\alpha_{j}\right|=m_{j}}\left\|D^{\alpha_{j}} b_{j}\right\|_{B M O}\right)\|f\|_{B_{p}(w) .}
\end{aligned}
$$

Thus

$$
L_{5} \leq C \prod_{j=1}^{2}\left(\sum_{\alpha_{j} \mid=m_{j}}\left\|D^{\alpha_{j}} b_{j}\right\|_{B M O}\right)\|f\|_{B_{p}(w) .}
$$

This finishes the proof of Theorem 2.

\section{Competing interests}

The authors declare that they have no competing interests.

\section{Authors' contributions}

The authors completed the paper. MZ carried out the ideas and methods of studies, YG participated in the design of the study and performed the statistical analysis, the sequence alignment and drafted the manuscript. The authors read and approved the final manuscript.

\section{Acknowledgements}

The work is supported by NNSFC (No. 11301097), Master Foundation of Guangxi University of Science and Technology (No. 0816208), Guangxi Education Institution Scientific Research Item (No. 2013YB170), GXNSF Grant (No. 2013GXNSFAA019001).

\section{Received: 1 June 2014 Accepted: 21 August 2014 Published: 03 Sep 2014}

\section{References}

1. Cohen, J: A sharp estimate for a multilinear singular integral on $R^{n}$. Indiana Univ. Math. J. 30, 693-702 (1981)

2. Cohen, J, Gosselin, J: On multilinear singular integral operators on $R^{n}$. Stud. Math. 72, 199-223 (1982)

3. Cohen, J, Gosselin, J: A BMO estimate for multilinear singular integral operators. III. J. Math. 30, 445-465 (1986)

4. Coifman, R, Meyer, Y: Wavelets, Calderón-Zygmund and Multilinear Operators. Cambridge Studies in Advanced Math., vol. 48. Cambridge University Press, Cambridge (1997)

5. Coifman, RR, Rochberg, R, Weiss, G: Factorization theorems for Hardy spaces in several variables. Ann. Math. 103, 611-635 (1976)

6. Garcia-Cuerva, J, Rubio de Francia, JL: Weighted Norm Inequalities and Related Topics. North-Holland Mathematics Studies, vol. 116. North-Holland, Amsterdam (1985)

7. Pérez, C, Pradolini, G: Sharp weighted endpoint estimates for commutators of singular integral operators. Mich. Math. J. 49, 23-37 (2001)

8. Pérez, C, Trujillo-Gonzalez, R: Sharp weighted estimates for multilinear commutators. J. Lond. Math. Soc. 65, 672-692 (2002)

9. Stein, EM: Harmonic Analysis: Real Variable Methods, Orthogonality and Oscillatory Integrals. Princeton University Press, Princeton (1993) 
10. Harboure, E, Segovia, C, Torrea, JL: Boundedness of commutators of fractional and singular integrals for the extreme values of $p$. III. J. Math. 41, 676-700 (1997)

11. Chang, DC, Li, JF, Xiao, J: Weighted scale estimates for Calderón-Zygmund type operators. Contemp. Math. 446, 61-70 (2007)

12. Lin, Y: Sharp maximal function estimates for Calderón-Zygmund type operators and commutators. Acta Math. Sci. Ser. A Chin. Ed. 31, 206-215 (2011)

13. Liu, LZ: Weighted boundedness of multilinear operators for the extreme cases. Taiwan. J. Math. 10, 669-690 (2006)

14. Liu, LZ: Weighted Herz type spaces estimates of multilinear singular integral operators for the extreme cases. Rev. R. Acad. Cienc. Exactas Fís. Nat., Ser. A Mat. 101, 87-98 (2007)

15. Liu, LZ: Endpoint estimates for multilinear fractional singular integral operators on some Hardy spaces. Math. Notes $88,735-752(2010)$

10.1186/1029-242X-2014-341

Cite this article as: Zhang and Guo: Weighted boundedness of multilinear singular integral operator with general

kernels for the extreme cases. Journal of Inequalities and Applications 2014, 2014:341

Submit your manuscript to a SpringerOpen ${ }^{\circ}$ journal and benefit from:

- Convenient online submission

Rigorous peer review

- Immediate publication on acceptance

- Open access: articles freely available online

- High visibility within the field

- Retaining the copyright to your article

Submit your next manuscript at $\gg$ springeropen.com 